Medical News

\title{
Potential 'universal' blood test for cancer discovered
}

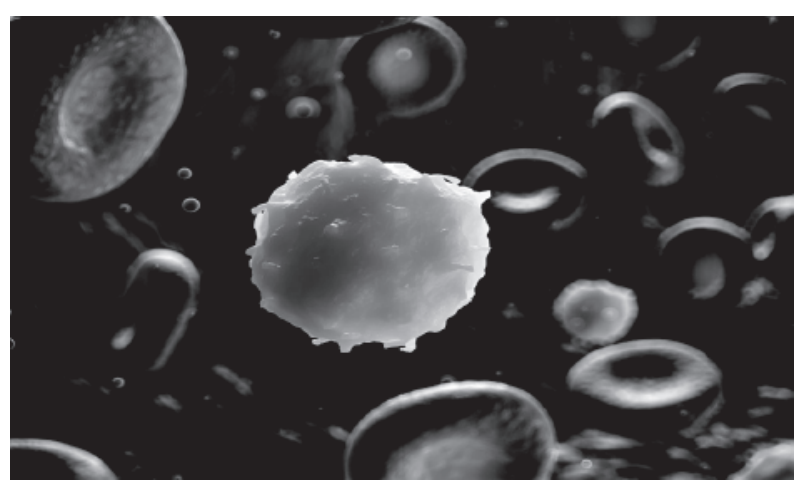

Researchers from the University of Bradford have devised a simple blood test that can be used to diagnose whether people have cancer or not. The test will enable doctors to rule out cancer in patients presenting with certain symptoms - saving time, and preventing costly and unnecessary invasive procedures such as colonoscopies and biopsies being carried out. Alternatively, it could be a useful aid for investigating patients who are suspected of having a cancer that is currently hard to diagnose.

Early results have shown the method gives a high degree of accuracy diagnosing cancer and pre cancerous conditions from the blood of patients with melanoma, colon cancer and lung cancer. The Lymphocyte Genome Sensitivity (LGS) test looks at white blood cells and measures the damage caused to their DNA when subjected to different intensities of ultraviolet light (UVA), which is known to damage DNA. The results of the empirical study show a clear distinction between the damage to the white blood cells from patients with cancer, with pre-cancerous conditions and from healthy patients.

The research was led by Professor Diana Anderson, from the University's School of Life Sciences, who says: "White blood cells are part of the body's natural defence system. We know that they are under stress when they are fighting cancer or other diseases, so I wondered whether anything measureable could be seen if we put them under further stress with UVA light. We found that people with cancer have DNA which is more easily damaged by ultraviolet light than other people, so the test shows the sensitivity to damage of all the DNA - the genome - in a cell." The study looked at blood samples taken from 208 individuals. Ninety-four healthy individuals were

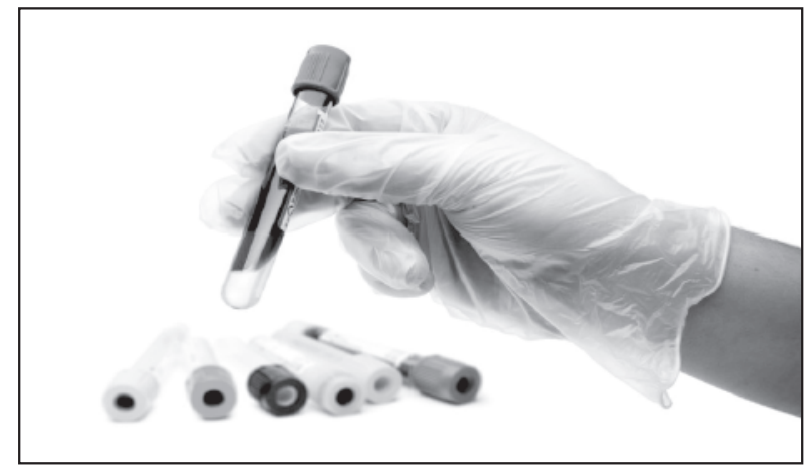

recruited from staff and students at the University and 114 blood samples were collected from patients referred to specialist clinics within Bradford Royal Infirmary prior to their diagnosis and treatment. The samples were coded, anonymised, randomised and then exposed to UVA light through five different depths of agar. UVA damage was observed in the form of DNA fragments being pulled in an electric field towards the positive end of the field, causing a comet-like tail. During the LGS test, the longer the tail the more DNA damage, and the measurements correlated to those patients who were ultimately diagnosed with cancer, those with pre-cancerous conditions and those who were healthy. "These are early results completed on three different types of cancer and we accept that more research needs to be done; but these results so far are remarkable," said Professor Anderson.

Professor Anderson believes that if the LGS proves to be a useful cancer diagnostic test, it would be a highly valuable addition to the more traditional investigative procedures for detecting cancer. A clinical trial is currently underway at Bradford Royal Infirmary. This will investigate the effectiveness of the LGS test in correctly predicting which patients referred by their GPs with suspected colorectal cancer would, or would not, benefit from a colonoscopy currently the preferred investigation method. The University of Bradford has filed patents for the technology and a spin-out company, Oncascan, has been established to commercialise the research.

Available from : www.futuretimeline.net/ blog/biologymedicine-blog.htm 